\title{
Feasibility of Using Improved Convolutional Neural Network to Classify BI-RADS 4 Breast Lesions: Compare Deep Learning Features of the Lesion Itself and the Minimum Bounding Cube of Lesion
}

\author{
Meihong Sheng $\left(\mathbb{D},{ }^{1,2}\right.$ Weixia Tang, ${ }^{2}$ Jiahuan Tang, ${ }^{2}$ Ming Zhang, ${ }^{2}$ Shenchu Gong, \\ and Wei Xing $\mathbb{D}^{1}$ \\ ${ }^{1}$ Department of Imaging, The Third Affiliated Hospital of Soochow University, Changzhou 213003, Jiangsu, China \\ ${ }^{2}$ Department of Imaging, Affiliated Hospital 2 of Nantong University, Nantong First People's Hospital, Nantong 226001, \\ Jiangsu, China \\ Correspondence should be addressed to Wei Xing; suzhxingwei@suda.edu.cn
}

Received 8 July 2021; Revised 3 August 2021; Accepted 6 August 2021; Published 8 September 2021

Academic Editor: Khin wee Lai

Copyright (c) 2021 Meihong Sheng et al. This is an open access article distributed under the Creative Commons Attribution License, which permits unrestricted use, distribution, and reproduction in any medium, provided the original work is properly cited.

\begin{abstract}
To determine the feasibility of using a deep learning (DL) approach to identify benign and malignant BI-RADS 4 lesions with preoperative breast DCE-MRI images and compare two 3D segmentation methods. The patients admitted from January 2014 to October 2020 were retrospectively analyzed. Breast MRI examination was performed before surgical resection or biopsy, and the masses were classified as BI-RADS 4. The first postcontrast images of DCE-MRI T1WI sequence were selected. There were two 3D segmentation methods for the lesions, one was manual segmentation along the edge of the lesion slice by slice, and the other was the minimum bounding cube of the lesion. Then, DL feature extraction was carried out; the pixel values of the image data are normalized to 0-1 range. The model was established based on the blueprint of the classic residual network ResNet50, retaining its residual module and improved $2 \mathrm{D}$ convolution module to $3 \mathrm{D}$. At the same time, an attention mechanism was added to transform the attention mechanism module, which only fit the $2 \mathrm{D}$ image convolution module, into a $3 \mathrm{D}$ Convolutional Block Attention Module (CBAM) to adapt to 3D-MRI. After the last CBAM, the algorithm stretches the output high-dimensional features into a one-dimensional vector and connects 2 fully connected slices, before finally setting two output results (P1, P2), which, respectively, represent the probability of benign and malignant lesions. Accuracy, sensitivity, specificity, negative predictive value, positive predictive value, the recall rate and area under the ROC curve (AUC) were used as evaluation indicators. A total of 203 patients were enrolled, with 207 mass lesions including 101 benign lesions and 106 malignant lesions. The data set was divided into the training set $(n=145)$, the validation set $(n=22)$, and the test set $(n=40)$ at the ratio of $7: 1: 2$; fivefold cross-validation was performed. The mean AUC based on the minimum bounding cube of lesion and the 3D-ROI of lesion itself were 0.827 and 0.799 , the accuracy was $78.54 \%$ and $74.63 \%$, the sensitivity was $78.85 \%$ and $83.65 \%$, the specificity was $78.22 \%$ and $65.35 \%$, the NPV was $78.85 \%$ and $71.31 \%$, the PPV was $78.22 \%$ and $79.52 \%$, the recall rate was $78.85 \%$ and $83.65 \%$, respectively. There was no statistical difference in AUC based on the lesion itself model and the minimum bounding cube model $(Z=0.771, p=0.4408)$. The minimum bounding cube based on the edge of the lesion showed higher accuracy, specificity, and lower recall rate in identifying benign and malignant lesions. Based on the lesion 3D-ROI segmentation using a minimum bounding cube can more effectively reflect the information of the lesion itself and the surrounding tissues. Its DL model performs better than the lesion itself. Using the DL approach with a 3D attention mechanism based on ResNet50 to identify benign and malignant BI-RADS 4 lesions was feasible.
\end{abstract}




\section{Introduction}

Breast cancer is a serious threat to women's health and has become the world's most common cancer [1]. Early detection, early diagnosis, and early treatment can improve both survival and prognosis of breast cancer patients [2-4]. Greenwood et al. [5] have reported that breast MRI plays an important role in screening and assessing the extent of ductal carcinoma in situ (DCIS) and predicting the potential invasiveness. The degree of early enhancement reflects the vascular richness and blood perfusion of the lesion and can reflect the characteristics of the lesion. According to the guideline of the American College of Radiology (ACR), the possibility range of the BIRADS 4 of malignancy is $2 \%-95 \%$ as defined by the breast imaging report and data system (BI-RADS) [6]. Lesions with BI-RADS 4 classification are difficult to define clearly. The signs of the lesions are overlapping and intricate. These lesions, benign or malignant, are all classified as BI-RADS 4, along with recommended invasive procedures such as needle biopsy to obtain pathological evidence [7-9]. Therefore, comprehensive understanding and improved evaluation methods of benign and malignant breast lesions are urgently needed to reduce invasive operations and the burden on patients.

In recent years, with the rapid development of artificial intelligence-assisted diagnosis systems, deep learning has emerged as a subfield of machine learning [10-13]. Its application in medical imaging has attracted much attention, along with its wide use in image recognition, segmentation, and analysis [14]. Several studies [15, 16] have attempted to increase the number of layers of CNNs from the original 5 layers of the AlexNet network [17] to the 19 layers of the VGG network. Theoretically, a deeper network leads to better effect, but the increase in network depth will also bring additional problems that in turn cause reduced performance. The main reason for the performance reduction was gradient dispersion (vanishing gradients in backpropagation lead to weakened error signal) and gradient explosion (accumulation of large error gradients results in infinity in loss function) that were caused by the increase in the number of network layers. The residual module was proposed by Khalili and Wong [15], which could effectively solve the aforementioned problems above and has become the standard configuration of CNNs.

The CNNs learned a large number of features. Some features were not important for the final result, while some others played a key role in predicting results thus deserve more attention. Based on this theory, Woo et al. [18] proposed the Convolutional Block Attention Module (CBAM). The so-called greater attention was to give higher weight to those key features. In this study, the efficiency of feature extraction and classification of BI-RADS 4 breast lesions with two segmentation methods was compared by the DL model with a 3D attention mechanism, so as to verify the feasibility of using an improved convolutional neural network.

\section{Materials and Methods}

2.1. Study Cohort and Imaging Protocol. The patients who underwent breast MRI examinations at Nantong First Peo- ple's Hospital were retrospectively collected from January 2014 to October 2020. A total of 296 patients with breast lesions were enrolled in the study. Inclusion criteria: (1) the diameter of the lesion was greater than $1 \mathrm{~cm}$, or lesions were visible to naked eyes at least two consecutive slices; (2) the image quality was high without obvious artifacts or distortion; (3) the lesions were all mass and showed irregular margins, or inhomogeneous enhancement, or ring enhancement in MRI and classified as BI-RADS 4 by the radiologist. Exclusion criteria: (1) the breast mass showed no enhancement; (2) radiotherapy/chemotherapy or invasive operations such as biopsy before breast MRI; (3) the characteristics of the lesion and the pathological diagnosis were not clear.

All MRIs in this study were acquired using a Siemens 3.0 $\mathrm{T}$ magnetic resonance scanner (Verio; Siemens, Erlangen, Germany) with 16-channel phased array breast-specific coil. The patients were placed in the prone position with headfirst entry; the breasts naturally hanged in the breast coil, and the nipple remained at the center of the coil. The scan sequence parameters were as follows: DCE T1-weighted axial fat suppression 3D spoiler gradient echo: TR $4.67 \mathrm{~ms}$, TE $1.66 \mathrm{~ms}$, flip angle $10^{\circ}$, FOV $340 \mathrm{~mm} \times 340 \mathrm{~mm}$, slice thickness $1.2 \mathrm{~mm}$, scanning of 6 phases without interval, scan time $6 \mathrm{~min} 25 \mathrm{~s}$, high-pressure syringe injection of 15$20 \mathrm{~mL}$ contrast agent Gd-DTPA based on body weight $(0.2 \mathrm{ml} / \mathrm{kg})$ at a flow rate of $2 \mathrm{~mL} / \mathrm{s}$, and then injection of the same amount of normal saline to flush the tube. After the $25 \mathrm{~s}$ injection, scanning was triggered, and each phase was collected for $1 \mathrm{~min}$. The first phase was nonenhancement, and phases 2-6 were enhanced. Our study focused on phase 2 images which was named DCE-MRI T1WI first postcontrast sequence.

2.2. 3D-ROI Lesion Segmentation. All DCE-MRI T1WI first postcontrast images of breast mass that meet the inclusion criteria were imported into the image processing software ITK-SNAP 3.8.0 in DICOM format, and the lesions were manually segmented by an attending physician with 8 years of experience in breast MRI diagnosis and reviewed by a chief physician with more than 10 years of experience in breast MRI diagnosis: (1) based on the ROI of the lesion itself (Figures 1 and 2), the 3D-ROI segmentation method was used to manually delineate the boundary of the lesion slice by slice along the edge of the lesion, containing cystic degeneration, necrosis, and calcification within the lesion; (2) based on the minimum bounding cube, the maximum diameter of the lesion was then projected onto 3 coordinate axes of the image to determine its coverage range of $x, y$, and $z$ axes, and the bounding box of the lesion was finally obtained (Figures 3 and 4).

2.3. Lesion Feature Extraction. There are two methods of feature extraction. One is to take the minimum bounding cube of the lesion (including the lesion and part of the peritumoral area), and the other is to take only the lesion itself and set the value of the image pixels of part of the nonlesion area to 0 . The minimum bounding cube is the smallest circumscribed cube containing the lesion. In addition, before 


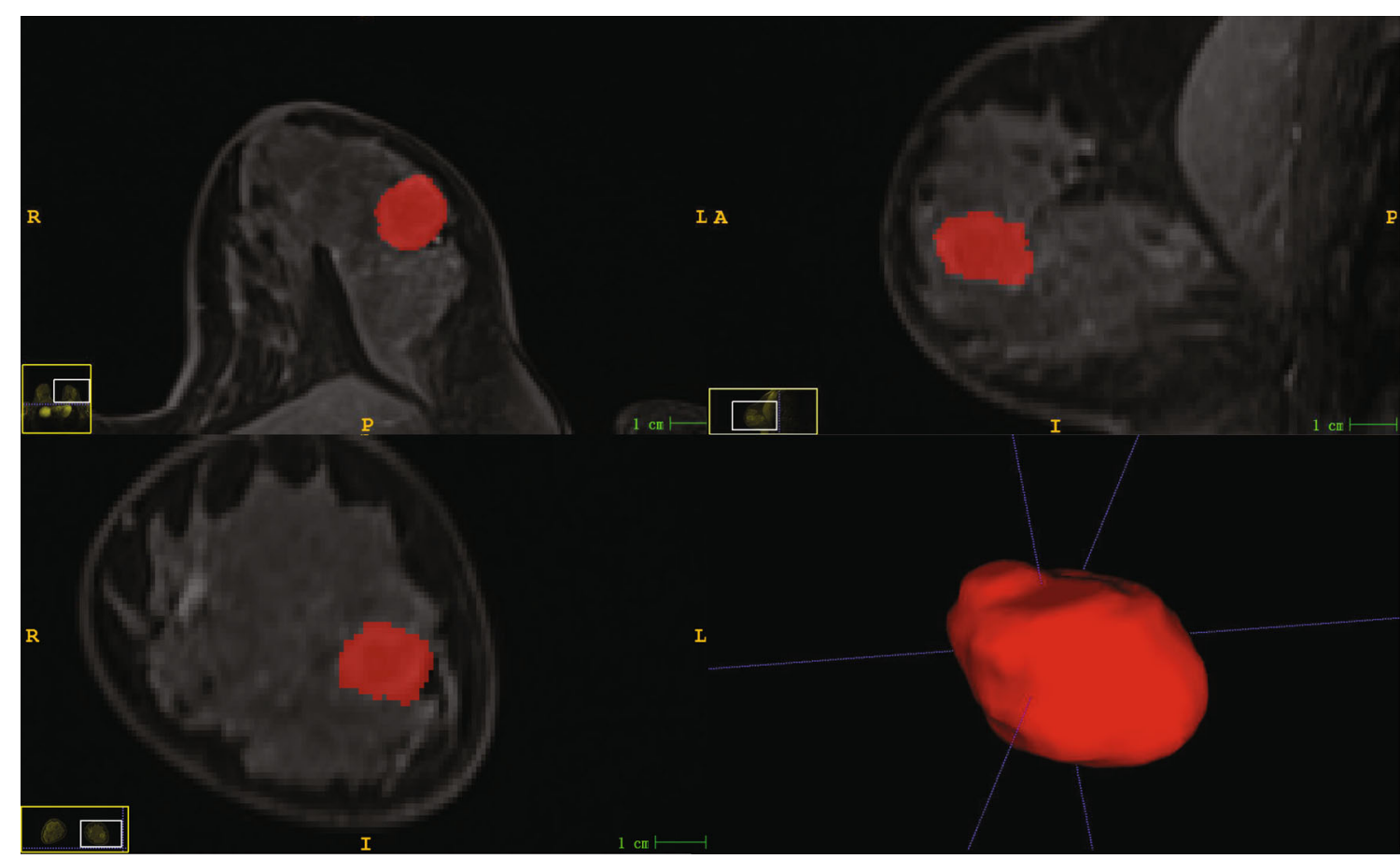

FIgURE 1: 3D-ROI segmentation method based on the lesion itself. Shows a case of fibroadenoma of the left breast.
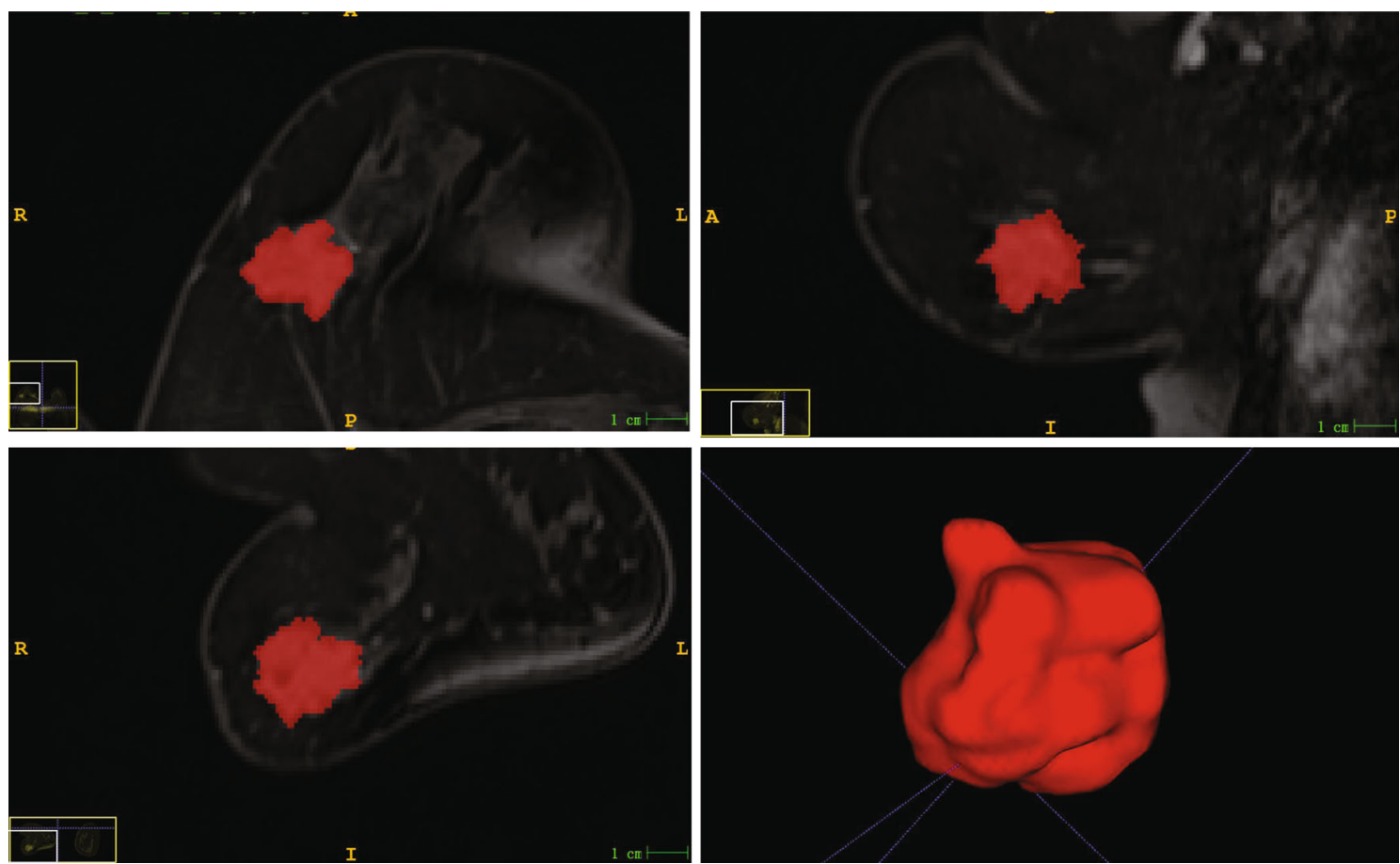

FIGURE 2: 3D-ROI segmentation method based on the lesion itself. Shows a case of invasive ductal carcinoma of the right breast (stage II).

inputting to the CNN, the pixel values of the image data are normalized to 0-1 range. The formula is as follows:

$$
x=\frac{X-X_{\min }}{X_{\max }-X_{\min }},
$$

where $x$ represents the normalized image pixel value, $X$ represents the original image pixel value, and $X_{\max }$ and $X_{\min }$ represent the maximum pixel value and the minimum pixel value of the minimum bounding cube of all lesions, respectively.

In this study, a total of 207 masses were obtained, of which 106 were malignant and 101 were benign. The data 


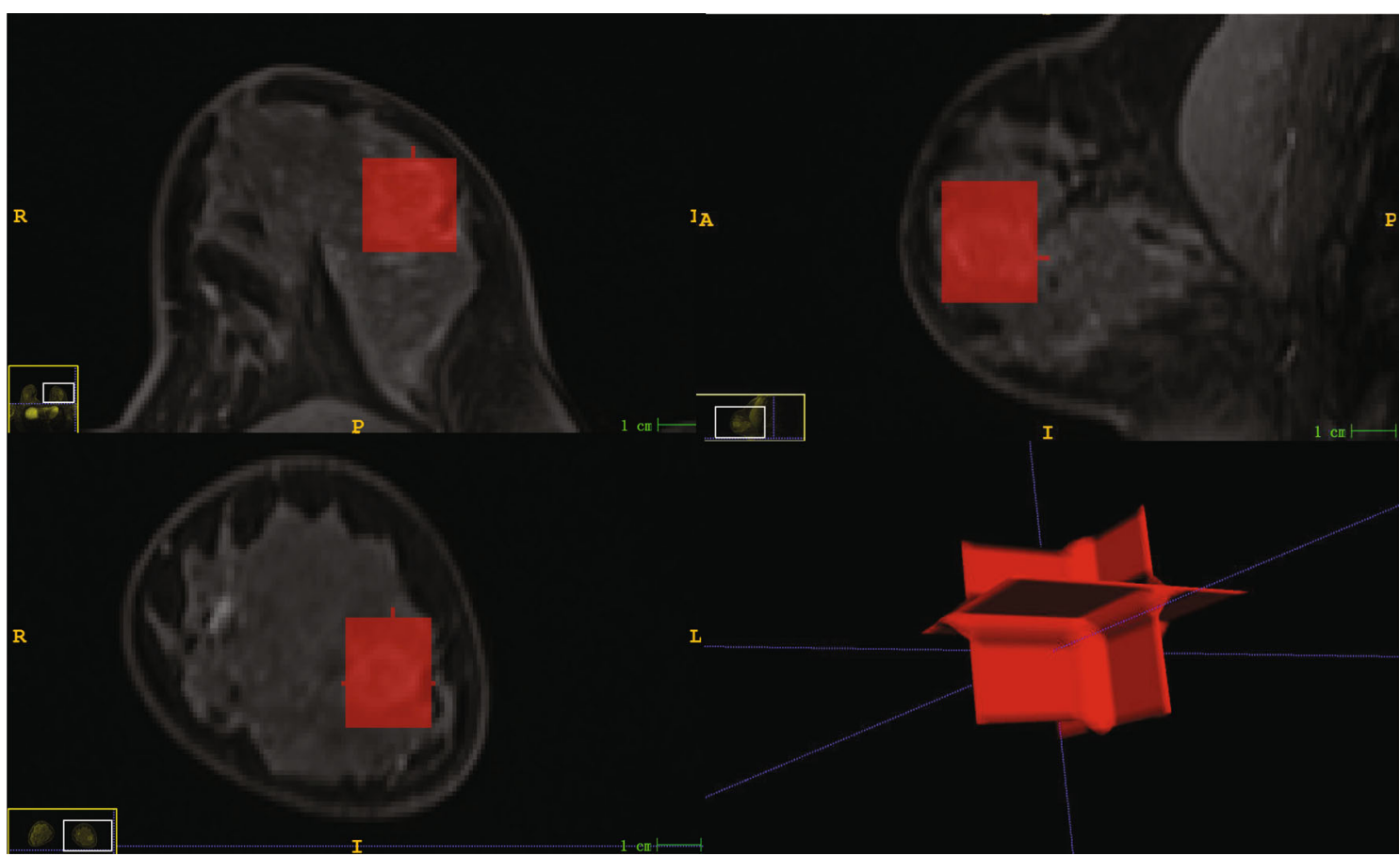

FIgURE 3: 3D-ROI segmentation method based on the minimum bounding cube at the edge of the lesion. Segmentation based on the minimum bounding box of the mass edge, showing a case of fibroadenoma of the left breast.

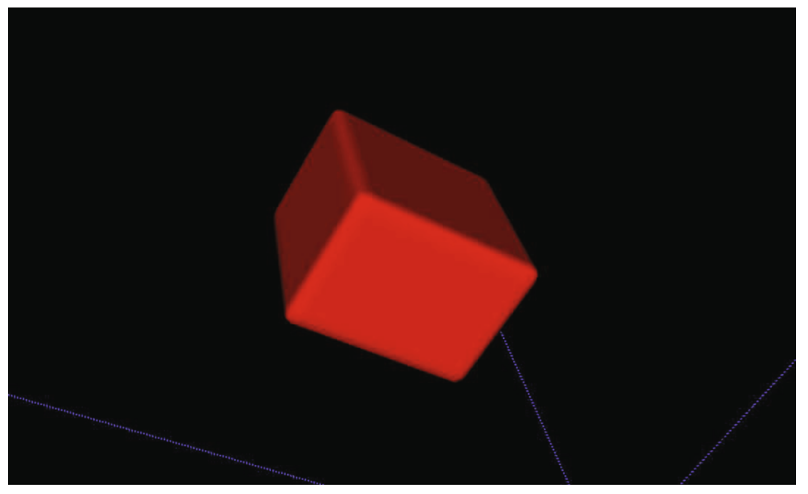

FIgURE 4: 3D-ROI segmentation method based on the minimum bounding cube at the edge of the lesion. The cubic model of segmentation based on the minimum bounding cube of the mass edge.

set was divided into the training set $(n=145)$, the validation set $(n=22)$, and the test set $(n=40)$ at the ratio of $7: 1: 2$. To avoid the selection bias of the benign and malignant lesions of the test set samples, the test set was generated by random sampling of 20 malignant lesions and 20 benign lesions. The remaining samples were randomly divided into the training set and the validation set in the ratio of $7: 1$. Fivefold crossvalidation was performed.

2.4. Model Establishment. The model was established based on the blueprint of the classic residual network ResNet50 [19], retaining its residual module but changing the convolution module to a $3 \mathrm{D}$ convolution module. At the same time, an attention mechanism was added to transform the attention mechanism module, which only fit the $2 \mathrm{D}$ image convolution module, into 3D-Convolutional Block Attention Module (CBAM) to adapt to 3D-MRI, as shown in Figure 5. CBAM includes a channel attention module and a spatial attention module, which together can solve the question of which channel and which position characteristics play decisive roles in final prediction [18]. Input module, residual module, channel attention module, downsampling module, and fully connected module constitute the main modules of the network. Among them, the residual module was mainly used to extract features, the CBAM module was mainly used to give higher weight to key features, and the downsampling module was used to reduce the size of the feature map and to increase the number of channels in the feature map. Blocks are used (Figure 5) to reflect the size change of the feature map. After the last CBAM, the algorithm stretches the output high-dimensional features into a one-dimensional vector and connects 2 fully connected slices. Lesion classification network parameters are shown in Table 1. The network uses cross-entropy cost function as the loss function and stochastic gradient descent (SGD) whose weight decay is 0.0001 and momentum is 0.9 as the optimizer. The batch size is 16 . Dynamic learning rate strategy is taken during the train process. The initial learning rate is 0.1 , which is considered as a big number, halved every 25 epochs of iterations. Before finally setting two output results $(\mathrm{P} 1, \mathrm{P} 2)$, which, respectively, represent the probability of benign and malignant lesions. The lesion is classified as benign if P1 > P2. Otherwise, the lesion is classified as malignant. 


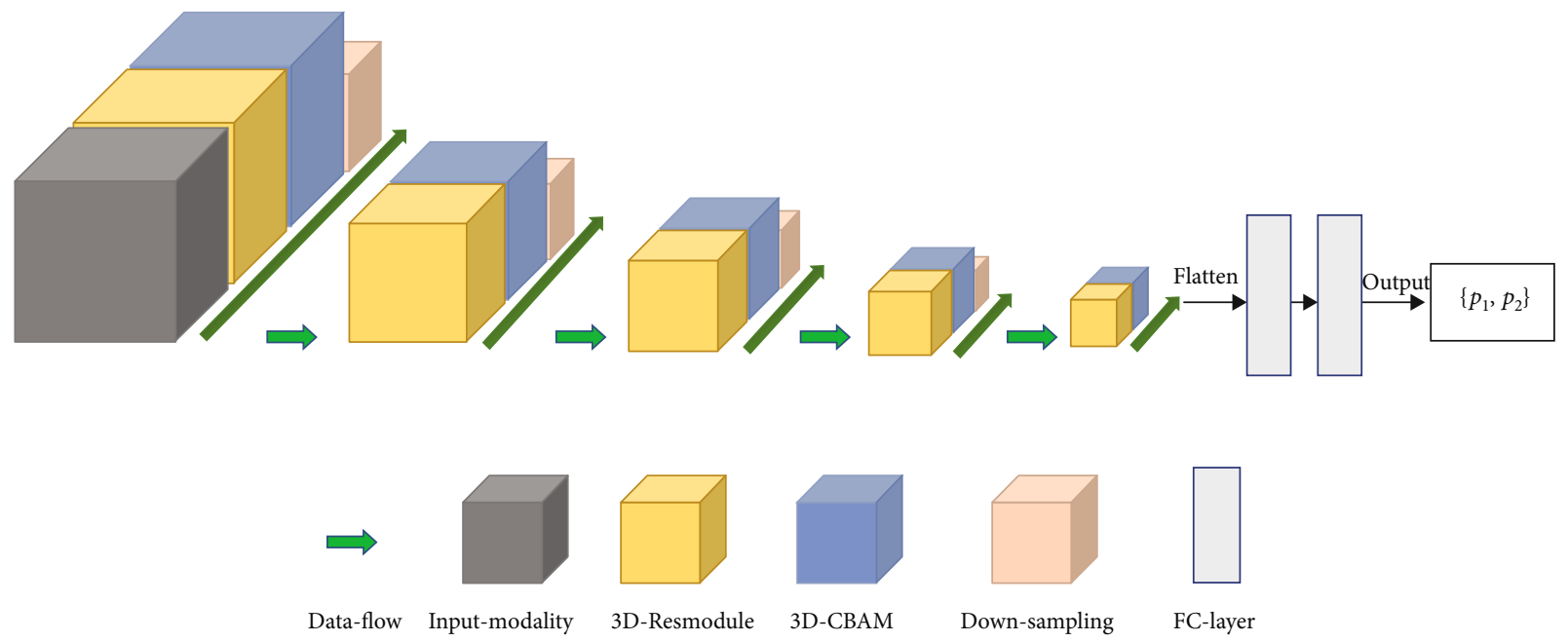

Figure 5: Structure of monomodal network.

TABLE 1: Tumor classification network parameters.

\begin{tabular}{|c|c|c|c|}
\hline Layer_name & Input_size & Detailed_parameters & Output_size \\
\hline conv & $1 * 64 * 64 * 64$ & Kernel $=3$, stride $=1$, padding $=1$ & $8 * 64 * 64 * 64$ \\
\hline res_conv $* 2$ & $8 * 64 * 64 * 64$ & Kernel $=3$, stride $=1$, padding $=1$ & $8 * 64 * 64 * 64$ \\
\hline 3D_CBAM & $8 * 64 * 64 * 64$ & $1 * 1 * 1$ convolution & $8 * 64 * 64 * 64$ \\
\hline conv & $8 * 64 * 64 * 64$ & Kernel $=2$, stride $=2$, padding $=0$ & $16 * 32 * 32 * 32$ \\
\hline res_conv $* 4$ & $16 * 32 * 32 * 32$ & Kernel $=3$, stride $=1$, padding $=1$ & $16 * 32 * 32 * 32$ \\
\hline 3D_CBAM & $16 * 32 * 32 * 32$ & $1 * 1 * 1$ convolution & $16 * 32 * 32 * 32$ \\
\hline conv & $16 * 32 * 32 * 32$ & Kernel $=2$, stride $=2$, padding $=0$ & $32 * 16 * 16 * 16$ \\
\hline res_conv $* 4$ & $32 * 16 * 16 * 16$ & Kernel $=3$, stride $=1$, padding $=1$ & $32 * 16 * 16 * 16$ \\
\hline 3D_CBAM & $32 * 16 * 16 * 16$ & $1 * 1 * 1$ convolution & $32 * 16 * 16 * 16$ \\
\hline conv & $32 * 16 * 16 * 16$ & Kernel $=2$, stride $=2$, padding $=0$ & $64 * 8 * 8 * 8$ \\
\hline res_conv $* 4$ & $64 * 8 * 8 * 8$ & Kernel $=3$, stride $=1$, padding $=1$ & $64 * 8 * 8 * 8$ \\
\hline 3D_CBAM & $64 * 8 * 8 * 8$ & $1 * 1 * 1$ convolution & $64 * 8 * 8 * 8$ \\
\hline conv & $64 * 8 * 8 * 8$ & Kernel $=2$, stride $=2$, padding $=0$ & $128 * 4 * 4 * 4$ \\
\hline res_conv $* 4$ & $128 * 4 * 4 * 4$ & Kernel $=3$, stride $=1$, padding $=1$ & $128 * 4 * 4 * 4$ \\
\hline 3D_CBAM & $128 * 4 * 4 * 4$ & $1 * 1 * 1$ convolution & $128 * 4 * 4 * 4$ \\
\hline Flatten & $128 * 4 * 4 * 4$ & & 8192 \\
\hline Full connection & 8192 & & 2048 \\
\hline Full connection & 2048 & & 512 \\
\hline Softmax & 512 & & 2 \\
\hline
\end{tabular}

"res_conv" is a residual convolution block which contains shortcut connection, and "res_conv $* N$ " means the block has $N$ convolution blocks that share the same parameters. 3D_CBAM uses $1 \times 1 \times 1$ convolutions to adjust the channel numbers of the current feature map.

2.5. Model Evaluation. The two DL feature models based on the lesion itself and the minimum boundary cube of lesion in the diagnosis of benign and malignant breast lesions were compared. Model performance was evaluated using the area under the ROC curve (AUC), accuracy, sensitivity, specificity, positive predictive value (PPV), negative predictive value (NPV), precision, and recall rate. The AUC of the two DL models was compared by the DeLong test.

\section{Results}

3.1. Patient Characteristics. A total of 296 patients with breast lesions were investigated in the study. The following cases were excluded: 3 patients with unqualified images due to the presence of motion artifacts, 12 patients with a history of biopsy or mastectomy before breast MRI examination, 53 patients with a single focal lesion or multiple focal 
TABLE 2: Age and pathological information of patients with benign and malignant breast tumors.

\begin{tabular}{lcc}
\hline & Benign patients $(n=98)$ & Malignant patients $(n=105)$ \\
\hline Age (year) & $41.0 \pm 10.6$ & $55.5 \pm 11.3$ \\
& Fibrocystic adenosis $(n=18)$ & Invasive ductal carcinoma $(n=81)$ \\
& Fibroadenoma $(n=71)$ & Mucinous adenocarcinoma of the breast $(n=7)$ \\
& Intraductal papilloma $(n=4)$ & Invasive lobular carcinoma $(n=3)$ \\
Pathology (207 masses) & Intraductal papillomatosis $(n=4)$ & Intraductal carcinoma or tubular carcinoma $(n=8)$ \\
& Benign phyllodes tumor $(n=4)$ & Solid papillary carcinoma $(n=2)$ \\
& & Malignant phyllodes tumor $(n=1)$ \\
& & Small lymphocytic lymphoma $(n=1)$ \\
& & Medullary carcinoma $(n=3)$
\end{tabular}

lesions in the breast, 14 patients with incomplete examination or perfusion scan breast MRI, and 11 patients with breast lesions combined with nonmass enhancement lesions. Eventually, 203 patients were enrolled for analyses (Table 1). The patients were 17-86 years old with an average age of $48.5 \pm 13.1$ years old. Among them, there was only one male patient, aged 54 years. There were 105 patients with malignant lesions with an average age of $55.5 \pm 11.3$ years and 98 patients with benign lesions with an average age of 41.0 \pm 10.6 years old. A total of 207 masses were included in the study (Table 2).

\subsection{Model Characteristics}

\subsubsection{Comparison of the Two Deep Learning Feature Model} Based on the Lesion Itself and the Minimum Boundary Cube of Lesion in the Diagnosis of Benign and Malignant Breast Lesions. Using first postcontrast images of DCEMRI T1WI sequence, the two deep learning feature model based on the lesion itself (model 1) and the minimum boundary cube of lesion (model 2) in the diagnosis of benign and malignant breast lesions were compared. The means are shown in Table 3. In comparison, the model 1 analysis achieved mean AUC of 0.799 , accuracy of $74.63 \%$, sensitivity of $83.65 \%$, specificity of $65.35 \%$, NPV of $71.31 \%$, PPV of $79.52 \%$, and recall rate of $83.65 \%$ and the model 2 analysis achieved an average AUC of 0.827 , accuracy of $78.54 \%$, sensitivity of $78.85 \%$, specificity and PPV of $78.22 \%$, NPV and recall rate of $78.85 \%$. There was no statistical difference in AUC based on the lesion itself model and the minimum bounding cube model $(Z=0.771, p=0.4408)$. The minimum bounding cube based on the edge of the lesion showed higher accuracy, specificity, and lower recall rate in identifying benign and malignant lesions.

\section{Discussion}

Deep learning in convolutional neural networks (CNNs) is usually based on manually or semiautomatically segmented tags to learn to recognize image features. Because breast MRI is different from MRI for abdomen and lung lesions, its position is fixed in a special breast coil and is less affected by breathing movement, leading to relatively higher reproducibility of the segmentation method for breast lesions.
TABLE 3: Comparison of two 3D-ROI segmentation methods based on the lesion itself and the minimum bounding cube at the edge of the lesion.

\begin{tabular}{lcc}
\hline Evaluation index & Lesion itself & Minimum bounding cube \\
\hline AUC & 0.799 & 0.827 \\
Accuracy (\%) & 74.63 & 78.54 \\
Sensitivity (\%) & 83.65 & 78.85 \\
Specificity (\%) & 65.35 & 78.22 \\
Negative prediction (\%) & 71.31 & 78.85 \\
Positive prediction (\%) & 79.52 & 78.22 \\
Precision (\%) & 71.31 & 78.85 \\
Recall (\%) & 83.65 & 78.85 \\
DeLong test & \multicolumn{2}{c}{$Z=0.771, p=0.4408$} \\
\hline
\end{tabular}

However, the segmentation methods are quite different. Previous studies have mostly extracted the two-dimensional features of the lesion (2D-ROI) [20], selected the largest slice of the lesion or the most obvious slice of lesion enhancement [21], and segmented along the edge of the lesion. 2D-ROI can only represent the information covered by the current area and cannot reflect all the information of the lesion. Therefore, this will definitely affect the reliability of DL models. The use of 3D-ROI is helpful to observe the lesion's overall morphology, leading to more accurate and comprehensive reflection of the characteristics of the lesion [22]. And more weight is given to the hemodynamic characteristics of the relevant lesion in the model based on the usual imaging physicians' reading habits and the advantages of early enhanced MRI.

4.1. The Efficacy of a Deep Learning Model Based on the Minimum Bounding Cube of the Lesion in Breast Lesion Classification. This study used two different segmentation methods for 3D-ROI of the lesion: one was based on the lesion itself, and the other one was based on the minimum bounding cube of the lesion edge. These two different segmentation methods were compared for their impact on the accuracy of the DL model. Our results revealed that the DL model based on the minimum bounding cube of the lesion edge is more accurate, with a mean AUC value of about 
0.827 . The reason may be that the minimum bounding cube based on the lesion edge not only contains the internal information of the lesion but also includes some tissues surrounding the lesion.

Zhou et al. [23] applied 5 different input boxes (tumor alone, the smallest bounding box, and 1.2, 1.5, and 2.0 time box) in deep learning and showed that the performance of diagnosis gradually decreases as the bounding box increases. The per-lesion diagnostic accuracy was the highest when using the smallest bounding box (89\%), but the tumor ROI on all slices were automatically segmented on contrastenhanced maps by using the fuzzy-C-means (FCM) clustering algorithm with $3 \mathrm{D}$ connected-component labeling, This study used manually segmented images as a standard for comparison, which may be more accurate. And the minimum bounding cube based on the tumor edge did not expand the box size but instead used 3D-CBAM to increase the weight of key information, in order to prevent the box containing too much information from normal tissue that dilutes the effective information in the overall box or reduces the resolution of the effective information of the image imported into the neural network.

The DL model that is based on the minimum bounding cube of postcontrast images of DCE-MRI T1WI sequence showed superiority in the test set, a mean specificity of $78.22 \%$, which are better than those of the DL model that is based on just the lesion itself. The reason may be that the microenvironment around the tumor plays a critical role in tumor growth and aggressive tissue behavior [24, 25]. 3DCBAM was to give higher weight to those key features. The area around the tumor contains much valuable and hidden information about the disease, including survival predictors for vascular activity and lymphangiogenesis and the infiltration of lymphatic and blood vessels around the tumor, and immune response signals around the tumor for interstitial response and lymphocyte infiltration around the tumor [26]. As we have shown in a previous study [27], the peritumoral edema on T2WI images is better and appears as T2WI hyperintensity around the tumor. This sign is combined with the T2WI signal, leading to significantly increased sensitivity and specificity for the differential diagnosis of benign and malignant breast tumors, and there is a positive correlation between peritumoral edema and Ki-67 expression. These results demonstrate the importance of the tissue surrounding the tumor. However, related studies are still limited at present; thus, the information about surrounding tissues has not been captured by the artificial intelligence learning technology. Braman et al. [26] collected a total of 117 patients and extracted omics features after marking the breast tumors and surrounding areas $(2.5-5 \mathrm{~mm}$ area around the tumor) using breast images from DCE-MRI-T1WI. Their results showed that the omics features of surrounding tissues helped to predict pCR and that combined use of tumor internal characteristics and peritumoral characteristics led to better prediction accuracy, which as a whole may help guide the personalized treatment of locally advanced breast cancer. This indicates that extracting the information contained in the tissue around the tumor has a high clinical application value.
4.2. The Diagnostic Efficacy of the Deep Learning Model Based on First Postcontrast Images of DCE-MRI T1WI Sequence in Benign and Malignant Breast Lesions. The deep learning model that is based on the minimum bounding cube of dynamic contrast postcontrast images has high specificity in the classification of benign and malignant breast lesions. We speculate that this may be related to the early hemodynamic information of the lesion, as shown in a previous study of ours that DCE-MRI can not only reveal tumor's morphological changes but also reflect its microvascular perfusion, angiogenesis, grades, and malignancy for evaluating the effect of tumor treatment and prognosis. The degree of early enhancement reflects the abundance of blood vessels and blood perfusion of the disease [28]. Malignant lesions grow fast, have multiple large blood vessels, are immature, and have a large number of arteriovenous anastomoses.

In addition to the high accuracy in diagnostic performance of the minimum bounding cube based on the edge of the lesion, we also found that the method is relatively simple and easy to use, as it only needs to find the largest level of the three dimensions of the image through image processing software. At this level, the minimum rectangle that can cover the outermost edge of the lesion is used, and finally, the minimum bounding cube containing the lesion is generated by the computer traversal method. However, the 3D-ROI based on the lesion itself needs to be delineated slice by slice and along the edge. For nonenhancement sequence images, sometimes, the edge of the lesion is unclear, leading to the lack of local edge information of the lesion.

\section{Conclusion}

In summary, based on the segmentation method of the minimum bounding cube at the edge of the lesion, postcontrast images of DCE-MRI T1WI sequence were extracted, and a DL model was established. This model can combine the information inside the lesion and that of containing peritumoral area to improve the diagnostic efficacy for both benign and malignant breast lesions. Using the DL approach with a $3 \mathrm{D}$ attention mechanism based on ResNet50 to identify benign and malignant BI-RADS 4 lesions was feasible.

\section{Limitations of This Study}

This study was a small-sample single-center study, and the results obtained in this study need to be confirmed by future large-sample multicenter investigations. Only mass lesions were included in the study; thus, whether the segmentation method is equally applicable to nonmass lesions remains to be tested. The inclusion/exclusion criteria are quite stringent and exclude many of the lesions which a radiologist reading breast MRI will routinely come across. The study only used first postcontrast images of DCE-MRI T1WI for segmentation by the minimum bounding cube of the lesion, which needs to be examined to see if it fits other sequences of image segmentation. Another limitation is that this study only compared two lesion segmentation methods; thus, future 
investigation is needed to test whether other ROIs containing peritumoral area may be better.

\section{Data Availability}

All data generated or analyzed during this study are available from the corresponding author Wei Xing upon reasonable request.

\section{Ethical Approval}

The retrospective study was approved by the Ethical Review Board of Nantong First People's Hospital (No. 2020KY236) and was conducted according to the Declaration of Helsinki principles.

\section{Consent}

All patients signed informed consent.

\section{Conflicts of Interest}

The authors declare that they have no conflict of interest.

\section{Authors' Contributions}

All authors made a significant contribution to the work reported, whether that is in the conception, study design, execution, acquisition of data, analysis, and interpretation or in all these areas; took part in drafting, revising, or critically reviewing the article; gave final approval of the version to be published; have agreed on the journal to which the article has been submitted; and agree to be accountable for all aspects of the work. Meihong Sheng and Weixia Tang are co-first authors.

\section{Acknowledgments}

This project was funded by the Jiangsu Province Maternal and Child Health Research Project (F202037), the "Six One" Research Funding Program for high-level health talents in Jiangsu Province (LGY2018036 and LGY2020048 in 2018 and 2020), and the Jiangsu Province Science and Technology Project (BE2018646).

\section{References}

[1] H. Sung, J. Ferlay, R. L. Siegel et al., "Global cancer statistics 2020: GLOBOCAN estimates of incidence and mortality worldwide for 36 cancers in 185 countries," CA: a Cancer Journal for Clinicians, vol. 71, no. 3, pp. 209-249, 2021.

[2] P. D. P. Pharoah, B. Sewell, D. Fitzsimmons, H. S. Bennett, and N. Pashayan, "Cost effectiveness of the NHS breast screening programme: life table model," BMJ, vol. 346, p. f2618, 2013.

[3] B. Lauby-Secretan, C. Scoccianti, D. Loomis et al., "Breast-cancer screening - viewpoint of the IARC working group," The New England Journal of Medicine, vol. 372, no. 24, pp. 23532358, 2015.

[4] M. G. Marmot, D. G. Altman, D. A. Cameron, J. A. Dewar, S. G. Thompson, and M. Wilcox, "The benefits and harms of breast cancer screening: an independent review," Lancet, vol. 380, pp. 1778-1786, 2012.

[5] H. I. Greenwood, L. J. Wilmes, T. Kelil, and B. N. Joe, "Role of breast MRI in the evaluation and detection of DCIS: opportunities and challenges," Journal of Magnetic Resonance Imaging, vol. 52, no. 3, pp. 697-709, 2020.

[6] E. A. Morris, C. E. Comstock, and C. H. Lee, ACR BI-RADS Magnetic Resonance Imaging, in: American College of Radiology, BI-RADS Committee, Editor. ACR BI-RADS Atlas: Breast Imaging Reporting and Data System, American College of Radiology, Reston, 5th edition, 2013.

[7] R. M. Strigel, E. S. Burnside, M. Elezaby et al., "Utility of BIRADS assessment category 4 subdivisions for screening breast MRI," AJR. American Journal of Roentgenology, vol. 2086, pp. 1392-1399, 2017.

[8] J. R. Maltez de Almeida, A. B. Gomes, T. P. Barros, P. E. Fahel, and M. de Seixas Rocha, "Subcategorization of suspicious breast lesions (BI-RADS category 4) according to MRI criteria: role of dynamic contrast-enhanced and diffusion-weighted imaging," AJR. American Journal of Roentgenology, vol. 205, no. 1, pp. 222-231, 2015.

[9] N. Houssami, S. Ciatto, I. Ellis, and D. Ambrogetti, "Underestimation of malignancy of breast core-needle biopsy: concepts and precise overall and category-specific estimates," Cancer, vol. 109, no. 3, pp. 487-495, 2007.

[10] K. Doi, "Computer-aided diagnosis in medical imaging: historical review, current status and future potential," Computerized Medical Imaging and Graphics, vol. 31, no. 4-5, pp. 198211, 2007.

[11] Y. Zhang, S. Wang, K. Xia, Y. Jiang, and P. Qian, “Alzheimer's disease multiclass diagnosis via multimodal neuroimaging embedding feature selection and fusion," Information Fusion, vol. 66, pp. 170-183, 2021.

[12] Y. Zhang, Z. Zhou, H. Bai, W. Liu, and L. Wang, "Seizure classification from EEG signals using an online selective transfer TSK fuzzy classifier with joint distribution adaption and manifold regularization," Frontiers in Neuroscience, vol. 14, p. 496, 2020.

[13] Y. Zhang, F. Chung, and S. Wang, "Clustering by transmission learning from data density to label manifold with statistical diffusion," Knowl Based Syst, vol. 193, p. 105330, 2020.

[14] A. Hosny, C. Parmar, J. Quackenbush, L. H. Schwartz, and H. J. W. L. Aerts, "Artificial intelligence in radiology," Nature Reviews. Cancer, vol. 18, no. 8, pp. 500-510, 2018.

[15] M. Khalili and R. J. Wong, "Underserved does not mean undeserved: unfurling the HCV care in the safety net," Digestive Diseases and Sciences, vol. 63, no. 12, pp. 32503252, 2018.

[16] C. Vasile, A. L. Udriștoiu, A. E. Ghenea et al., "Intelligent diagnosis of thyroid ultrasound imaging using an ensemble of deep learning methods," Medicina (Kaunas), vol. 57, no. 4, p. 395, 2021.

[17] A. Krizhevsky, I. Sutskever, and G. E. Hinton, "Imagenet classification with deep convolutional neural networks," Advances in Neural Information Processing Systems, vol. 25, pp. 10971105, 2012.

[18] S. Woo, J. Park, J. Y. Lee, and I. S. Kweon, "CBAM: convolutional block attention module," European Conference on Computer Vision (ECCV), pp. 3-19, 2018.

[19] S. M. Anwar, M. Majid, A. Qayyum, M. Awais, M. Alnowami, and M. K. Khan, "Medical image analysis using convolutional 
neural networks: a review," Journal of Medical Systems, vol. 42, no. 11, p. 226, 2018.

[20] P. Herent, B. Schmauch, P. Jehanno et al., "Detection and characterization of MRI breast lesions using deep learning," Diagnostic and Interventional Imaging, vol. 100, no. 4, pp. 219-225, 2019.

[21] K. Holli-Helenius, A. Salminen, I. Rinta-Kiikka et al., "MRI texture analysis in differentiating luminal A and luminal B breast cancer molecular subtypes - a feasibility study," BMC Medical Imaging, vol. 17, no. 1, p. 69, 2017.

[22] C. Song, W. S. Zhu, S. Y. Shi et al., "Differentiation between benign and malignant non-mass enhancement lesions using volumetric quantitative dynamic contrast-enhanced MR imaging," Radiology Practice, vol. 35, no. 2, pp. 190-196, 2020.

[23] J. Zhou, Y. Zhang, K. T. Chang et al., "Diagnosis of benign and malignant breast lesions on DCE-MRI by using radiomics and deep learning with consideration of peritumor tissue," Journal of Magnetic Resonance Imaging, vol. 51, no. 3, pp. 798-809, 2020.

[24] Y. Kim, M. A. Stolarska, and H. G. Othmer, "The role of the microenvironment in tumor growth and invasion," Progress in Biophysics and Molecular Biology, vol. 106, no. 2, pp. 353379, 2011.

[25] J. S. Wu, S. R. Sheng, X. H. Liang, and Y. L. Tang, "The role of tumor microenvironment in collective tumor cell invasion," Future Oncology, vol. 13, no. 11, pp. 991-1002, 2017.

[26] N. M. Braman, M. Etesami, P. Prasanna et al., "Intratumoral and peritumoral radiomics for the pretreatment prediction of pathological complete response to neoadjuvant chemotherapy based on breast DCE-MRI," Breast Cancer Research, vol. 19, no. 1, p. 57, 2017.

[27] W. X. Tang, M. H. Sheng, S. C. Gong, H. T. Chen, J. B. Ge, and Y. Zhu, "Diagnostic value of T2WI relative signal intensity ratio combined with peritumoral edema in benign and malignant breast lesions," Journal of Clinical Radiology, vol. 39, no. 12, pp. 2411-2414, 2020.

[28] M. H. Sheng, W. X. Tang, Y. H. Lu et al., "Value of early-phase enhancement ratio combined with peripheral vascular diameter in the differential diagnosis of benign and malignant breast lesions under dynamic contrast enhanced MRI," Chinese Journal of Radiology, vol. 50, no. 5, pp. 324-328, 2016. 